\title{
Lealdade do Consumidor e Programas de Fidelidade: UMA ANÁLISE TOPOGRÁFICA DO CAMPO DE CONHECIMENTO À LUZ DA BIBLIOMETRIA, DA ESTATÍSTICA ESPACIAL E DAS REDES SOCIAIS
}

\author{
Consumer Loyalty and Loyalty Programs: a topographic \\ examination of the scientific literature using bibliometrics, \\ spatial statistics and network analyses
}

\author{
Viviane Moura Rocha \\ Mestre em Administração de Empresas. FGV-EAESP. São Paulo, SP. Brasil. E-mail: vivianemr@bol.com.br \\ Mateus Canniatti Ponchio \\ Doutor em Administração de Empresas. ESPM e FGV-EAESP. São Paulo, SP. Brasil.E-mail: mponchio@espm.br
}

Eduardo de Rezende Francisco

Doutor em Administração de Empresas. ESPM e FGV-EAESP. São Paulo, SP. Brasil. E-mail: erfrancisco@gmail.com

\section{Resumo}

Apresenta-se neste artigo uma análise topográfica do campo de conhecimento constituído pelos conceitos de lealdade do consumidor e pelos programas de fidelidade, amplamente estudados nas últimas décadas e ainda relevantes à área de marketing. A partir de 250 artigos acadêmicos publicados nos últimos dez anos em periódicos científicos indexados, selecionouse um subconjunto de 76 que continham 3.223 artigos acadêmicos referenciados. Foram tabulados $e$ analisados os periódicos de publicação, as palavraschave, os resumos, os autores, suas instituições de origem $e$ as redes de citação correspondentes por meio de técnicas bibliométricas, estatística espacial e análise de redes. Os resultados permitem identificar a centralidade desses componentes no campo e os principais autores, periódicos, instituições e países intermediadores da difusão do conhecimento, o que favorece a compreensão da constituição do campo por pesquisadores e estudantes.

Palavras-chave: Lealdade. Programas de Fidelidade. Bibliometria. Estatística Espacial. Análise de Redes.

\section{Abstract}

This paper presents a topographic analysis of the fields of consumer loyalty and loyalty programs, vastly studied in the last decades and still relevant in the marketing literature. After the identification of 250 scientific papers that were published in the last ten years in indexed journals, a subset of 76 were chosen and their 3.223 references were extracted. The journals in which these papers were published, their key words, abstracts, authors, institutions of origin and citation patterns were identified and analyzed using bibliometrics, spatial statistics techniques and network analyses. The results allow the identification of the central components of the field, as well as its main authors, journals, institutions and countries that intermediate the diffusion of knowledge, which contributes to the understanding of the constitution of the field by researchers and students.

Keywords: Loyalty. Loyalty Programs. Bibliometrics. Spatial Statistics. Network Analyses. 


\section{INTRODUÇÃo}

Nas últimas décadas, milhares de estudos teóricos e empíricos que envolvem conceitos de lealdade do consumidor no ambiente varejista foram publicados. O conceito de lealdade do consumidor ocupa posição central no marketing e, como campo de conhecimento, se mantém de interesse primário na academia. (HARDESTY; BEARDEN, 2009)

A conceituação de lealdade provém de uma abordagem comportamental, em que o comportamento de compra repetitivo era central (FRANK, 1967); passa por uma abordagem cognitiva, com foco nas dimensões atitudinais do construto (DAY, 1969), e chega a uma abordagem composta, de acordo com a qual comportamento e atitude são duas condições essenciais à lealdade (DICK; BASU, 1994). Oliver (1999) e McMullan e Gilmore (2003) propuseram, ainda, abordagens processuais - com destaque às fases de cognição, de afeição, de conação e de ação - para conceituar lealdade.

Apesar da quantidade de estudos disponíveis, há diversidade, se não divergência, de conceituação e de operacionalização do construto lealdade, de seus antecedentes e de seus consequentes (TOUFAILY; RICARD; PERRIEN, 2012). Pesquisas na área enfatizam lealdade a uma marca ou produto, a uma unidade de varejo ou a uma organização. A lealdade é também frequentemente avaliada em contextos específicos, como por exemplo, na esfera de serviços quando associada a programas de relacionamento.

Recentes esforços de sistematizar a produção da área merecem destaque. Toufaily, Ricard e Perrien (2012) conduziram uma meta-análise de estudos empíricos, baseada em 44 artigos, e propuseram um modelo integrativo com cinco componentes antecedentes da lealdade (características do consumidor, características do ambiente, atributos dos produtos e serviços, características da empresa/do varejista, e características do website) para medir o construto em contextos de e-commerce. Pan, Sheng e Xie (2012), em outra meta-análise sobre os antecedentes da lealdade, propuseram três componentes: satisfação do consumidor, valor percebido e tipo de produto. Yoo e Bai (2013), em um extenso trabalho de revisão do campo de conhecimento, analisaram 262 artigos sobre lealdade do consumidor e os classificaram quanto às áreas e setores pesquisados, conceituações e métodos empregados. Entre os achados, constatou-se que as abordagens comportamental e atitudinal estão fortemente presentes (em conjunto) nos estudos, com alguma variação no peso atribuído a cada uma delas; que o método quantitativo de análise de dados obtidos em surveys é o mais empregado; e que as técnicas de análise de modelagem por equações estruturais $e$ modelos lineares e não lineares são as mais frequentes.

A discussão sobre lealdade do consumidor e do marketing num sentido mais amplo vem sendo feita primariamente em periódicos científicos, e o número de periódicos relacionados à área, bem como o volume de artigos acadêmicos neles publicados, cresceu rapidamente nas últimas décadas (BAUMGARTNER; PIETERS, 2003). Independentemente de o campo ser familiar ou não a um pesquisador, os grandes volumes de informação disponíveis, bem como a dispersão da informação, tornam as atividades de coleta e análise complexas e tempo-intensivas. Inclusive, já há quase duas décadas Garfield (1996) havia notado que o dinamismo com que a produção de conhecimento se propaga impede que a velocidade de progresso científico se iguale à velocidade de disseminação de artigos acadêmicos em geral. É razoável supor que esse cenário esteja agravado atualmente.

Justamente por ser um tema central à área de marketing, já bastante explorado, o campo de conhecimento originado dos conceitos de lealdade do consumidor e marketing de relacionamento torna-se objeto relevante de análise para este trabalho. Nosso objetivo é mapear a estrutura desse campo, da origem das produções ao padrão de interligação do conhecimento.

Os objetivos específicos são: (i) identificar os artigos e periódicos do núcleo desse campo de conhecimento; (ii) identificar seus principais pesquisadores, grupos e instituições; (iii) estudar a dispersão da literatura disponível; (iv) medir o grau e padrões de colaboração entre autores; e (v) analisar processos de citação e cocitação com a finalidade de identificar a contribuição de autores predecessores para o estado da arte do campo. Entende-se que as referências utilizadas por um autor, além de servirem como suporte de argumentação, evidenciam as influências de conceitos e metodologias passadas em seu trabalho.

Os resultados deste tipo de estudo são de utilidade prática para pesquisadores, educadores e estu- 
dantes em geral (BAUMGARTNER; PIETERS, 2003) e complementam os esforços de sistematização da contribuição substantiva da área que são feitos por meio de meta-análises.

Para atingir esses objetivos, apresenta-se, nas seções seguintes, as revisões dos conceitos abordados, a metodologia de pesquisa, os resultados das análises quantitativas, e, por fim, as considerações finais.

\section{Lealdade do Consumidor e Programas de Fidelidade}

Estudar o consumidor, em particular suas ações no ambiente de varejo, é crítico para que estratégias varejistas possam ser desenvolvidas e implementadas de maneira efetiva (LEVY; WEITZ, 2007). As empresas reconhecem a importância da lealdade como fator influenciador de vendas e de aumento de market share (ZEITHAML, 2000). Em análise realizada por Narayandas (2005), foi identificado que $80 \%$ das empresas monitoram a lealdade dos seus clientes. Essa lealdade mensurada e almejada pelas empresas significa aumento de tempo de relacionamento, que pode ser traduzida em maior receita por cliente. A proposta inicial dos programas de fidelidade, de maneira geral, é promover ou aumentar essa fidelidade de consumo desejada e por isso a importância de estudá-los. Uma linha bastante desenvolvida e relevante de investigação acadêmica envolve o estudo de questões referentes à influência dos mecanismos do programa de fidelidade no comportamento do consumidor e, consequentemente, em sua lealdade. (ANGELO; FAVERO; EUNNI, 2007; LIANG; MA; QI, 2012; ALONSO-ALMEIDA; BREMSER, 2013)

Segundo Marques e Brasil (2008), o entendimento principal que se deve ter do conceito de lealdade é sua dupla personalidade, baseada não só em aspectos comportamentais, mas, principalmente, em aspectos atitudinais. Esses autores baseiam-se na interpretação de lealdade oferecida por Dick e Basu (1994), que é a mais citada nos artigos estudados neste trabalho (Tabela 5). Outras interpretações de lealdade foram encontradas em artigos identificados na pesquisa de campo conduzida para o presente estudo e estão relatadas no Quadro 1.

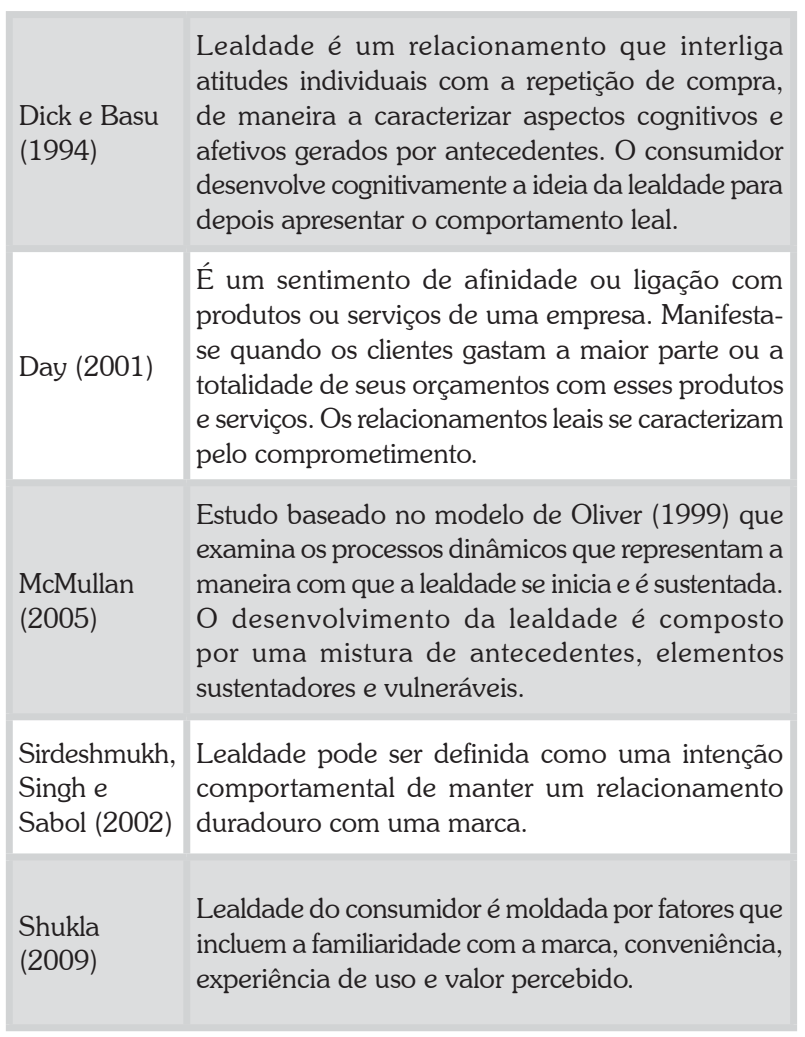

Quadro 1: Concepção de lealdade segundo autores identificados na pesquisa de campo

Fonte: Elaborado pelos autores deste artigo

No ambiente virtual, algumas definições de lealdade do consumidor são: probabilidade de visitas repetidas por um mesmo indivíduo (ARMSTRONG; HAGEL, 1996), intenção de fazer novas compras em um mesmo website no futuro (JOHNSON; BRUNER; KUMAR, 2006) e atitude favorável do consumidor perante o negócio virtual que resulta em compras repetidas (HUANG, 2008; RABBANEE et al., 2012). Cabe ressaltar a diferente ênfase em componentes comportamentais e atitudinais dessas definições.

Segundo Oliveira, Toledo e Ikeda (2004), um dos principais objetivos dos programas de fidelização é aumentar o nível de retenção de clientes, pois à medida que são oferecidos benefícios adicionais aos clientes fiéis, eles se colocam como barreiras à troca da empresa de consumo. Além disso, os programas de fidelidade podem ser usados também como ferramenta para conhecer os clientes: podem ser coletados dados a respeito do consumidor que, posteriormente analisados, servem de base para ações mercadológicas, melhorias de atendimento e busca de eficiências operacionais 
diversas. Na década de 1990, com o boom tecnológico, os dados coletados nos programas, em conjunto com o desenvolvimento de ferramentas com recursos de database marketing, permitiram a realização de análises ainda mais elaboradas a respeito dos clientes (CAPIZZI; FERGUSON, 2005). Esse avanço permitiu às empresas conhecer melhor o perfil dos clientes utilizando os seus próprios recursos. Assim, além de as empresas criarem formas de recompensar os clientes, também constroem bancos de dados robustos para melhor entendê-los.

Ao avaliar programas de fidelidade, devem ser levados em consideração os conceitos de benefícios monetários e não monetários. Segundo Chandon, Wansink e Gilles (2000), os benefícios monetários podem ser definidos como benefícios de economia, qualidade superior e conveniência. Já os benefícios não monetários, segundo os mesmos autores, são associados a status e entretenimento. Segundo Melnyk e Osselaer (2012), os benefícios não monetários, denominados pelo autor de benefícios psicológicos, são importantes para os programas de fidelidade, pois são menos dispendiosos que os benefícios monetários.

Na formulação dos programas de fidelidade, segundo Nunes e Drèze (2006), alguns objetivos em relação ao comportamento do consumidor são pré-estabelecidos: criar uma barreira à saída, concentrar gastos, gerar informações sobre consumo às empresas, $e$ incrementar a lucratividade e o volume de compras adicionais.

Os programas de fidelidade se tornaram amplamente conhecidos na década de 1980, por meio das companhias aéreas e cartões de crédito, fundamentalmente (BERMAN, 2006). Segundo Rocha e Veloso (1999), o primeiro programa de fidelidade baseado no conceito de viajante frequente foi criado em 1980 pela American Airlines, chamado AAdvantage. Pela primeira vez foi criada uma estrutura com banco de dados para contabilizar os pontos dos afiliados do programa de fidelidade. Tal mecanismo demorou anos para ser adotado pela concorrência, o que concedeu à companhia vantagem competitiva durante o período.

Em 2010, o número de membros na soma dos programas de fidelidade de empresas norte-americanas ultrapassou dois bilhões (HLAVINKA; SULLIVAN, 2011). No mercado brasileiro é sabido que existem inúmeros programas de relacionamento em atuação nos mais diversos ramos. Segundo notícia veiculada no Portal Folha de São Paulo (2013), os programas de fidelidade no Brasil apresentam números bastante expressivos. Como exemplo, o Multiplus Fidelidade, programa criado pela companhia aérea TAM e que consolida pontos acumulados pelo consumo de produtos e serviços de várias empresas, possui mais de 10,5 milhões de associados e o programa Smiles, da empresa aérea GOL, mais de 9 milhões de clientes. (PORTAL FOLHA DE SÃO PAULO, 2013)

\section{Procedimentos Metodológicos}

Existem diversas formas de avaliação do conhecimento científico e de medição de fluxos de informação. Para esse propósito, a ideia de indexar citações e outros elementos de artigos acadêmicos data do final do século XIX, mas apenas na década de 1950 é que métodos computacionais para automatizar essa rotina começaram a ser desenvolvidos (GARFIELD, 1979). Neste trabalho, são adotadas técnicas bibliométricas - mais tradicionais -, e algumas técnicas de análise de redes e de análise espacial de disseminação mais recente.

A bibliometria é o estudo dos aspectos quantitativos da produção, disseminação e uso da informação registrada com emprego de métodos matemáticos e estatísticos (SPINAK, 1996). Já as técnicas de análise de redes permitem desenhar as redes de modo a melhor comunicar centralidades ou relacionamentos entre seus elementos geralmente em duas ou três dimensões (BRANDES; KENIS; WAGNER, 2003). Essas redes espacialmente representadas tornam-se "georreferenciadas", ou seja, seus elementos (nós e arestas) adquirem caracterização espacial, o que habilita o uso de praticamente todo o ferramental da análise espacial. (FRANCISCO, 2011)

\subsection{Escolha das Bases de Dados}

Por possuir uma das maiores bases de artigos acadêmicos na área de negócios disponível atualmente, o portal ScienceDirect / Scopus (disponível em: <www. sciverse.com >) foi eleito como ponto de partida para o estudo. Durante o primeiro trimestre de 2013, foram localizados todos os artigos acadêmicos publicados em periódicos científicos que: (i) continham os termos loyalty program ou loyalty programs; (ii) haviam sido publicados entre 2004 e 2013; e (iii) continham a palavra-chave customer loyalty. Alguns artigos publica- 
dos em outros idiomas (como por exemplo, espanhol e alemão) foram descartados em função de limitações das técnicas de análise para associar o conteúdo de seus textos com o conteúdo dos textos de língua inglesa.

Foram coletados 250 artigos acadêmicos. Elaborou-se um banco de dados que continha campos específicos para armazenar, de cada um desses artigos: título, periódico de veiculação, ano de publicação, sobrenome e iniciais do nome de cada um dos autores e coautores, afiliação de cada autor e coautor (instituição e país), além do resumo (na íntegra). Foram feitas extensas checagens de digitação e de similaridade de termos para evitar duplicidades ou inconsistências, principalmente nos sobrenomes dos autores.

Os periódicos com mais artigos veiculados foram os seguintes (em parênteses a quantidade): Journal of Retailing and Consumer Services (18), Journal of Consumer Marketing (17), Journal of Services Marketing (11), Journal of Business Research (10), Journal of Retailing (10) e International Journal of Hospitality Management (10). Os demais 174 artigos estavam diluídos em 109 diferentes periódicos. A partir das referências daqueles 76 artigos, foram filtradas apenas as referentes a artigos acadêmicos publicados em periódicos científicos. A filtragem foi manual. O total de registros desse segundo banco de dados foi 3.223. Novamente, uma ampla checagem de consistência dos dados foi executada.

\section{Análises e Resultados}

A análise de palavras foi iniciada com uma preparação dos resumos dos 250 artigos. Palavras estruturais (stop words: artigos, verbos de ligação, pontuação, números, alguns adjetivos) foram removidas e os principais termos foram convertidos para o singular (por exemplo, customer e customers). Em seguida, as palavras foram contabilizadas.

As 20 palavras mais citadas foram selecionadas, $e$ as coocorrências em um mesmo artigo foram observadas. Uma matriz de coocorrências foi construída, pontuando a diversidade de artigos (dos 250) em que cada duas palavras ocorriam simultaneamente. Adicionalmente, a diagonal dessa matriz foi pontuada de acordo com a diversidade de artigos em que cada palavra, isoladamente, ocorria.

A partir da matriz de coocorrências, uma rede de associações entre as palavras mais frequentes foi construída. A Figura 1 apresenta essa rede, com tamanhos dos vértices (palavras) proporcionais à quantidade de artigos em que cada palavra ocorreu. A rede possui densidade máxima (1), o que significa que todas as palavras coocorrem em pelo menos um dos 250 artigos.

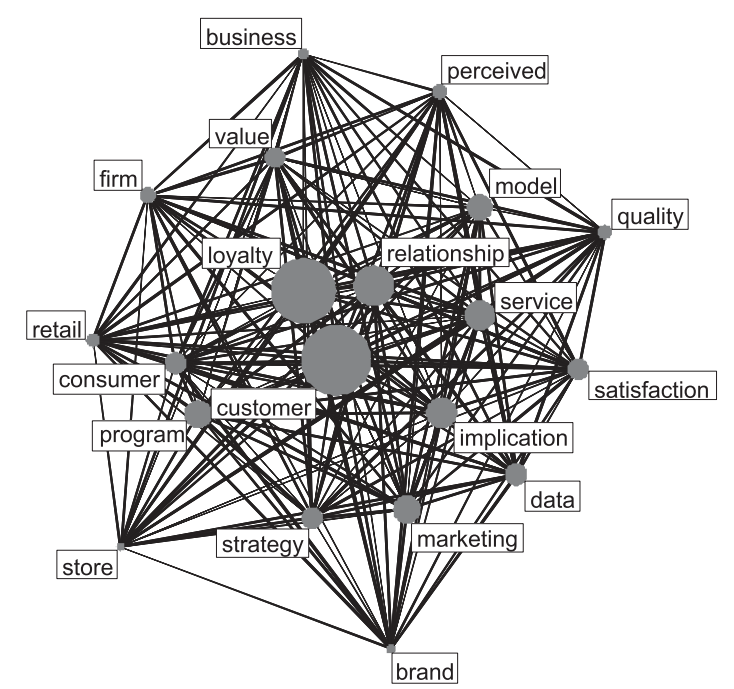

Figura 1: Rede de coocorrências das 20 palavras mais frequentes dos 250 artigos analisados

Fonte: Elaborada pelos autores deste artigo com uso da ferramenta R 2.15 .2 (extensão network)

Representando apenas as coocorrências em pelo menos 20 artigos, procurando destacar, dessa forma, as palavras com mais associação, notou-se (conforme Figura 1 e Figura 2) que as palavras customer, loyalty e relationship apresentam posições centrais na rede, o que é corroborado por suas grandes centralidades de intermediação, de grau e sua maior diversidade de ocorrência dentre os 250 artigos. A Tabela 1 apresenta esses valores para as 20 palavras-chave. A densidade dessa rede, que exclui as arestas de menor representatividade de associação, é 50,52\%.

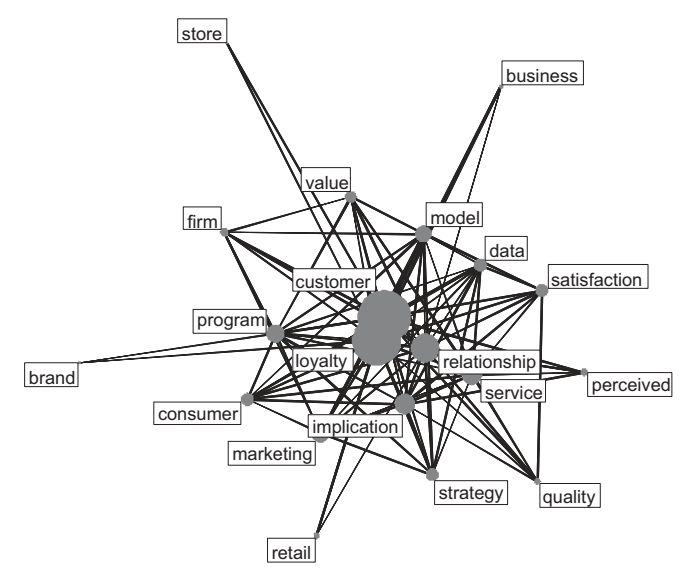

Figura 2: Rede de coocorrências (mínimo de 20 artigos) das 20 palavras mais frequentes dos 250 artigos analisados Fonte: Elaborada pelos autores deste artigo com uso da ferramenta R 2.15 .2 (extensão network) 
Tabela 1: Estatísticas descritivas da rede de coocorrências das 20 palavras mais frequentes nos resumos dos 250 artigos analisados

\begin{tabular}{|c|c|c|c|c|c|c|c|}
\hline Palavra & $\begin{array}{c}\text { FREQUÊNCIA DE } \\
\text { COOCORRÊNCIA } \\
\text { COM AS DEMAIS } \\
19 \text { PALAVRAS }\end{array}$ & $\begin{array}{c}\text { CENTRALIDAdE } \\
\text { DE } \\
\text { INTERMEDIAÇÃo }\end{array}$ & $\begin{array}{c}\text { DIVERSIDADE } \\
\text { DE ARTIGOS } \\
\text { EM QUE } \\
\text { OCORREU }\end{array}$ & Palavia & $\begin{array}{c}\text { FREQUÊNCIA DE } \\
\text { COOCORRÊNCIA } \\
\text { COM AS DEMAIS } \\
19 \text { PALAVRAS }\end{array}$ & $\begin{array}{c}\text { Centralidade } \\
\text { DE } \\
\text { INTERMEDIAÇÃo }\end{array}$ & $\begin{array}{c}\text { DiversidADE } \\
\text { DE ARTIGOS } \\
\text { EM QUE } \\
\text { OCORREU }\end{array}$ \\
\hline Customer & 1326 & 31,74 & 225 & strategy & 469 & 0,00 & 70 \\
\hline Loyalty & 1259 & 31,74 & 210 & value & 445 & 0,29 & 66 \\
\hline relationship & 843 & 15,24 & 131 & firm & 343 & 0,13 & 53 \\
\hline program & 558 & 1,45 & 90 & data & 484 & 0,68 & 72 \\
\hline service & 657 & 4,54 & 98 & brand & 204 & 0,00 & 31 \\
\hline satisfaction & 466 & 0,33 & 69 & implication & 675 & 4,54 & 99 \\
\hline marketing & 576 & 0,90 & 90 & store & 191 & 0,00 & 28 \\
\hline consumer & 458 & 0,00 & 71 & perceived & 349 & 0,00 & 49 \\
\hline Model & 553 & 2,44 & 84 & business & 236 & 0,00 & 37 \\
\hline Quality & 323 & 0,00 & 46 & retail & 299 & 0,00 & 45 \\
\hline
\end{tabular}

Fonte: Elaborada pelos autores deste artigo

Após a realização de análise similar de coocorrência das 20 palavras mais frequentes apenas dos 76 artigos analisados mais detalhadamente, construiu-se a rede de coocorrências, conforme Figura 3. Essa rede apresenta também altíssima densidade $(95,8 \%)$.

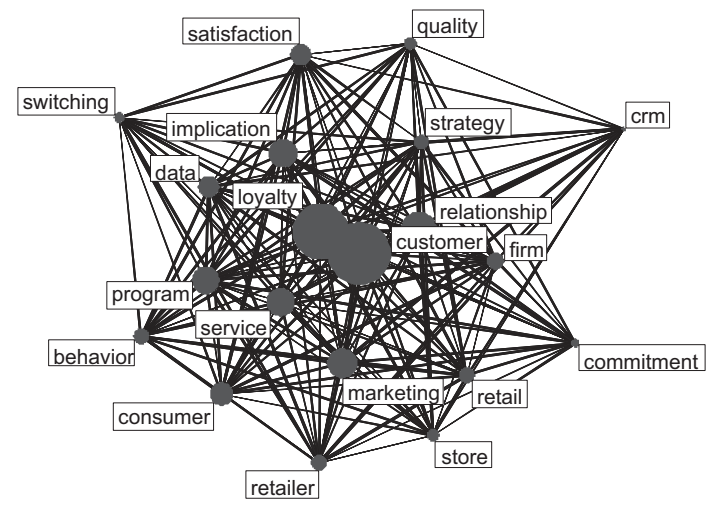

Figura 3: Rede de coocorrências das 20 palavras mais frequentes dos 76 artigos analisados

Fonte: Elaborada pelos autores deste artigo com uso da ferramenta R 2.15.2 (extensão network)

Nota-se similaridade muito grande entre as palavras mais frequentes do conjunto de 250 artigos e de seu subconjunto de 76 artigos. Tal similaridade sugere que uma análise de frequência e associação de palavras pode se dar satisfatoriamente em apenas um subconjunto representativo do acervo analisado, conforme abordagem desenvolvida neste estudo.
A observação das palavras mais representativas e suas posições de destaque na rede são plenamente obtidas a partir de um subconjunto de artigos.

\subsection{Análise de Autoria e Afiliação de Autores}

A Tabela 2 apresenta o número de autores por artigo, para cada um dos 250 artigos da base inicial.

Tabela 2: Distribuição de frequência de número de autores por artigo

\begin{tabular}{|c|c|c|c|}
\hline $\begin{array}{c}\text { NÚMERO DE } \\
\text { AUTORES POR } \\
\text { ARTIGO }\end{array}$ & $\begin{array}{c}\text { NÚMERO DE } \\
\text { ARTIGOS }\end{array}$ & $\begin{array}{c}\text { NÚMERO DE } \\
\text { AUTORES POR } \\
\text { ARTIGO }\end{array}$ & $\begin{array}{c}\text { NúMERO DE } \\
\text { ARTIGOS }\end{array}$ \\
\hline 1 & 41 & 4 & 25 \\
\hline 2 & 89 & 5 & 7 \\
\hline 3 & 84 & 6 ou mais & 4 \\
\hline
\end{tabular}

Fonte: Elaborada pelos autores deste artigo

Os 250 artigos foram escritos em coautoria por 348 instituições. As instituições estão organizadas em 168 grupos de colaboração, dos quais 81 são os nós isolados - representam instituições que não colaboraram em coautoria com outras. A grande quantidade de componentes dessa rede indica baixa colaboração 
entre as instituições. Desses grupos, destacam-se dois com 11 instituições e um com 10. A Figura 4 representa a rede de colaboração entre instituições.

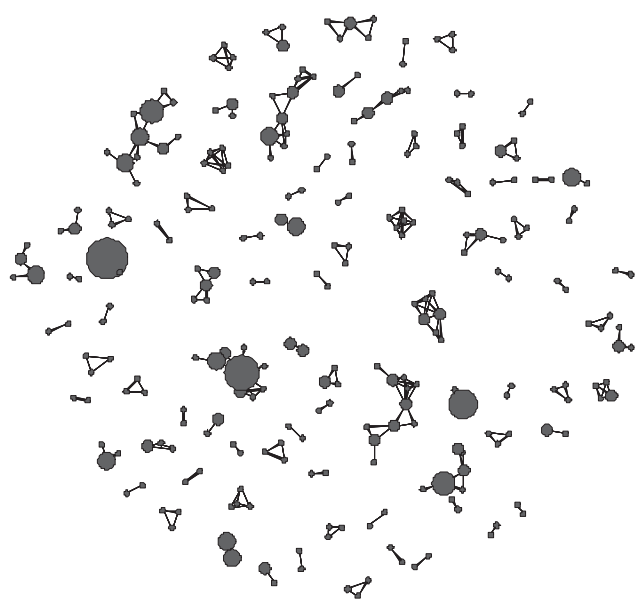

Figura 4: Rede de colaboração entre instituições Fonte: Elaborada pelos autores deste artigo com uso da ferramenta R 2.15.2 (extensão network)

É possível destacar em maior colaboração em coautorias entre instituições a Universiti Teknologi MARA, da Malásia (11 colaborações), a University of Groningen, da Noruega e Holanda (11 colaborações) e a Curtin University, da Austrália (nove colaborações).

Os 250 artigos foram escritos por instituições de 45 países. Trinta e dois dos 45 países escreveram em coautoria. Um componente dessa rede apresenta 29 países em colaboração, conforme destaque na Figura 5. Outros dois componentes apresentam dois países e os demais 14 componentes foram escritos por instituições de um mesmo país.

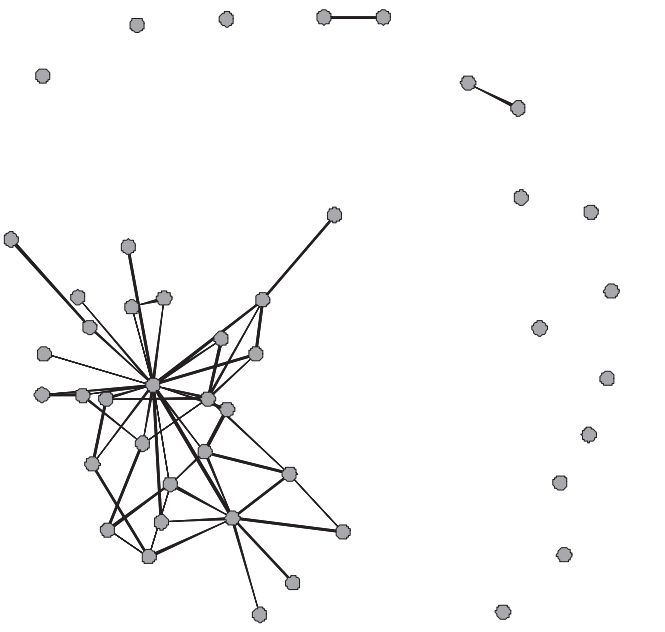

Figura 5: Rede de colaboração entre países das instituições dos 250 artigos analisados (principal componente da rede em destaque) Fonte: Elaborada pelos autores deste artigo com uso das ferramentas ArcGIS 10.1, extensões 3D Analyst e Spatial Analyst
Tabela 3: Países com maior número de artigos publicados

\begin{tabular}{|c|c|c|c|}
\hline País & $\begin{array}{c}\text { ARTI- } \\
\text { GOS }\end{array}$ & $\begin{array}{c}\text { Centralida- } \\
\text { DE DE GRAu } \\
\text { (ARTIGOS EM } \\
\text { COLABORAÇÃo) }\end{array}$ & $\begin{array}{c}\text { CENTRALidAdE DE } \\
\text { InTERMEDIAÇÃo }\end{array}$ \\
\hline EUA & 96 & 79 & 281,03 \\
\hline Alemanha & 15 & 26 & 8,60 \\
\hline Canadá & 11 & 24 & 20,73 \\
\hline Reino Unido & 17 & 21 & 87,13 \\
\hline China & 16 & 16 & 11,83 \\
\hline Austrália & 15 & 13 & 16,23 \\
\hline Áustria & 3 & 11 & 0,00 \\
\hline Holanda & 8 & 10 & 6,45 \\
\hline Nova Zelândia & 5 & 9 & 1,92 \\
\hline Noruega & 3 & 5 & 6,37 \\
\hline
\end{tabular}

Fonte: Elaborada pelos autores deste artigo

A Tabela 3 traz os países com maior número de artigos, maior centralidade de intermediação (betweenness centrality), medida da intermediação de uma instituição nas relações com as demais, servindo como ponte para a interação entre elas (HANNEMAN; RIDDLE, 2005), e maior centralidade de grau (artigos em colaboração). Destacam-se EUA, Reino Unido, Alemanha e Canadá como os países com maior importância na disseminação $e$ fluidez das informações no campo de estudo da lealdade, a partir do acervo de 250 artigos selecionados para esta análise.

Optou-se por trabalhar com a componente principal da rede para as análises de centralidade, conforme sugerem Newman (2001) e Moody (2004). A partir da análise da principal componente da rede, foram construídos modelos de superfície a partir de técnicas de estatística espacial (FOWLER, 1976). As posições e distâncias entre os 29 países nessa rede foram obtidos a partir de sua colaboração na rede (BUTTS, 2008; BUTTS; HANDCOCK; HUNTER, 2011), e suas qualificações quanto a centralidade de intermediação e de grau foram utilizadas como pesos (alturas) nos modelos de superfície. Apesar da menor quantidade de artigos em colaboração do que Alemanha e Canadá, o Reino Unido destaca-se por maior intermediação. 


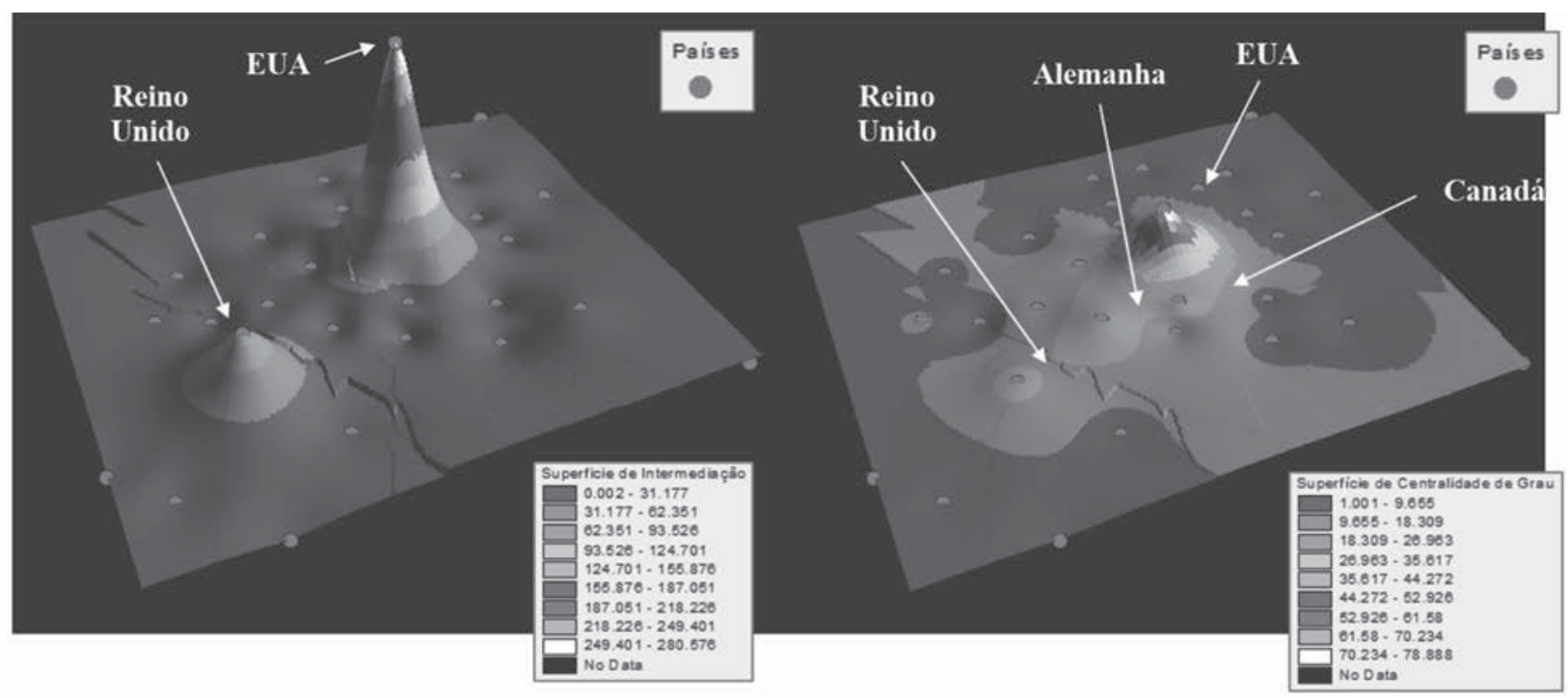

Figura 6: Superfícies de Intermediação (esq.) e de Centralidade de Grau (dir.) da principal componente de países das instituições em colaboração em coautorias dos 250 artigos analisados

Fonte: Elaborada pelos autores deste artigo por meio das ferramentas ArcGIS 10.1, extensões 3D Analyst e Spatial Analyst

Os 250 artigos foram escritos por 480 diferentes autores. Foram 452 autores que escreveram apenas um artigo, 21 com dois artigos, quatro com três artigos, dois com quatro artigos e apenas um autor (K. Hlavinka) com cinco artigos no acervo completo analisado. Apenas 28 autores, pois, escreveram mais de um artigo, o que sugere pouca colaboração na coautoria, apesar de a média de autores por artigo ter sido 2,54. Corroborando a descrição anterior, a análise da rede de coautorias mostra que a colaboração entre autores neste acervo foi relativamente pequena. 222 componentes foram identificados - uma densidade de rede de apenas $0,32 \%$. 31 componentes isolados (31 autores que escreveram um único artigo, sem coautoria); 152 componentes com dois autores; e 39 componentes com pelo menos três.

\subsection{Análise de Referências}

Dos 76 artigos escolhidos para a segunda rodada de análises, foram extraídas 3.223 referências a artigos científicos publicados em periódicos. Os autores mais referenciados são:
Tabela 4: Principais autores citados nas 3.223 referências bibliográficas dos 76 artigos analisados

\begin{tabular}{|c|c|c|c|}
\hline \multicolumn{1}{|c|}{$\begin{array}{c}\text { QuANTIDADE } \\
\text { Autor }\end{array}$} & $\begin{array}{c}\text { DE } \\
\text { REFERÊNCIAS }\end{array}$ & Autor & $\begin{array}{c}\text { QuANTIDADE } \\
\text { DE } \\
\text { REFERÊNCIAS }\end{array}$ \\
\hline $\begin{array}{c}\text { Valerie A. } \\
\text { Zeithaml }\end{array}$ & 285 & E. W. Anderson & 56 \\
\hline $\begin{array}{c}\text { Leonard } \\
\text { L. Berry }\end{array}$ & 282 & Ruth N. Bolton & 49 \\
\hline $\begin{array}{c}\text { V. Kumar } \\
\text { Richard L. } \\
\text { Oliver }\end{array}$ & 144 & Mark D. Uncles & 46 \\
\hline $\begin{array}{c}\text { Kristy Ellis } \\
\text { Reynolds }\end{array}$ & 60 & R. Anderson & 44 \\
\hline
\end{tabular}

Fonte: Elaborada pelos autores deste artigo

A Tabela 5, embora apresente a vantagem de identificar, a partir dos artigos do próprio campo de conhecimento, quais são aqueles mais referenciados (este seria um ranking de certa forma mais qualificado do que um ranking geral de citações na academia), possui a limitação de favorecer, talvez, aqueles artigos publicados há mais tempo e que, por isso, têm maior chance de ser citados por artigos publicados de 2004 a 2013. A Tabela 6 apresenta a relação dos periódicos científicos mais referenciados. 
Tabela 5: Relação de artigos acadêmicos mais referenciados

\begin{tabular}{|c|c|c|c|c|}
\hline Título & $\begin{array}{c}\text { Ano de } \\
\text { Publicação }\end{array}$ & Periódico & Autores & $\begin{array}{c}\text { ARTIGOS QUE O } \\
\text { CITARAM (DOS 76) }\end{array}$ \\
\hline $\begin{array}{l}\text { Customer loyalty: toward an } \\
\text { integrated conceptual framework }\end{array}$ & 1994 & $\begin{array}{l}\text { Journal of the Academy } \\
\text { of Marketing Science }\end{array}$ & A.S. Dick; K. Basu & 24 \\
\hline $\begin{array}{l}\text { Do customer loyalty } \\
\text { programs really work? }\end{array}$ & 1997 & Sloan Management Review & G.R. Dowling; M. Uncles & 22 \\
\hline Whence consumer loyalty? & 1999 & Journal of Marketing & R.L. Oliver & 22 \\
\hline $\begin{array}{l}\text { Loyalty programs and their impact } \\
\text { on repeat purchase loyalty patterns }\end{array}$ & 1997 & $\begin{array}{l}\text { International Journal of } \\
\text { Research in Marketing }\end{array}$ & B. Sharp; A. Sharp & 19 \\
\hline $\begin{array}{l}\text { Implications of loyalty program } \\
\text { membership and service experiences } \\
\text { for customer retention and value }\end{array}$ & 2000 & $\begin{array}{l}\text { Journal of the Academy } \\
\text { of Marketing Science }\end{array}$ & $\begin{array}{l}\text { R.N. Bolton; P.K. } \\
\text { Kannan; M.D. Bramlett }\end{array}$ & 18 \\
\hline $\begin{array}{l}\text { The commitment-trust theory } \\
\text { of relationship management }\end{array}$ & 1994 & Journal of Marketing & R.M. Morgan; S.D. Hunt & 18 \\
\hline
\end{tabular}

Fonte: Elaborada pelos autores deste artigo

Tabela 6: Relação de periódicos científicos mais referenciados (entre as 3.223 referências)

\begin{tabular}{|c|c|c|c|}
\hline Periódico & FrEQUÊNCIA & Periódico & FrEQUÊNCIA \\
\hline Journal of Marketing & 394 & Journal of Service Research & 83 \\
\hline Journal of Retailing & 394 & Journal of Consumer Research & 82 \\
\hline $\begin{array}{l}\text { Journal of the Academy } \\
\text { of Marketing Science }\end{array}$ & 195 & $\begin{array}{l}\text { Journal of Retailing and } \\
\text { Consumer Services }\end{array}$ & 82 \\
\hline Journal of Marketing Research & 185 & $\begin{array}{l}\text { International Journal of } \\
\text { Research in Marketing }\end{array}$ & 66 \\
\hline Journal of Business Research & 109 & Journal of Services Marketing & 64 \\
\hline Harvard Business Review & 106 & Journal of Consumer Marketing & 56 \\
\hline
\end{tabular}

Fonte: Elaborada pelos autores deste artigo

\section{Considerações Finais}

A proposta desse estudo foi desenvolver uma investigação analítica de artigos acadêmicos acerca dos conceitos de lealdade e programas de fidelidade para representar a topografia do campo. O uso combinado de técnicas convencionais de bibliometria com as análises de redes sociais e técnicas de estatística espacial permitiu um entendimento da estrutura $e$ das características de relacionamento entre autores, instituições e entre assuntos.

Especificamente, foram identificados os artigos e periódicos do núcleo desse campo de conhecimento (Tabelas 5 e 6, respectivamente) e seus principais pesquisadores, grupos e instituições (Tabela 4 e Figuras 4 e 5). As análises apresentadas permitiram identificar os centros de pesquisa (instituições, países e autores) mais influentes sobre lealdade e programas de fidelidade, além de produzir rankings de autores e periódicos científicos mais citados.

Quanto à dispersão da literatura disponível (Figura 4), ao grau e ao padrão de colaboração entre os autores (Tabela 2 e Figuras 4, 5 e 6), vale destacar a baixa colaboração na autoria dos artigos (medida por meio de coautorias), ou, talvez, a grande pulverização - institucional e geográfica - de centros de pesquisa acerca do campo de conhecimento. Dentre 250 artigos acadêmicos acerca de lealdade do consumidor e programas de fidelidade, foram identificadas 348 instituições participantes dessa produção científica, organizadas em torno do elevado número de 168 redes de colaboração. É possível, por outro lado, que esse resultado seja explicado justamente pela relevância do 
tema, que conta com artigos científicos desenvolvidos por instituições de dezenas de países. Pode-se, de qualquer maneira, imaginar saudável ao desenvolvimento substantivo do campo que ocorram mais parcerias e intercâmbio entre pesquisadores, que poderiam ser refletidas nas redes de colaboração.

Finalmente, de maneira mais ampla, foram investigados os processos de citação e cocitação com o propósito de identificar a contribuição de autores predecessores para o estado da arte do campo. Sobre este último objetivo específico, sugere-se que, em futuros estudos, seja incorporada a variável tempo à construção das redes de citação e cocitação; dessa forma, o índice 'citações por período de tempo' poderia ser incorporado à análise, contribuindo para destacar a relevância de artigos que passam a ser muito citados desde o momento em que são publicados.

Certamente, as análises aqui endereçadas podem ser reexecutadas a partir de bancos de dados maiores que 250 ou 3.223 registros. A opção por analisar apenas artigos de periódicos foi motivada pela simplificação e pela percepção de centralidade desse tipo de veículo no campo de conhecimento. No entanto, certamente são possíveis e possuirão méritos análises que englobem outros tipos de publicação, tais como artigos de congressos e livros. Serão também bem-vindas técnicas de análise que permitam comparar conteúdos de artigos acadêmicos publicados em diferentes idiomas. No tocante ao conteúdo do campo de conhecimento, em editorial do Journal of Retailing, Hardesty e Bearden (2009) propõem, como possibilidade de novas pesquisas, investigar o relacionamento entre consumidores e varejistas e avaliar a influência na lealdade da percepção daqueles quanto à justiça $e$ à legitimidade de ofertas de varejo mais vantajosas a um grupo de consumidores do que a outro.

As análises de redes de citação basearam-se nos 3.223 registros provenientes de 76 artigos acadêmicos (dos 250 originalmente identificados). Pode-se indagar se as descobertas referentes a artigos, autores e instituições mais influentes teriam sido as mesmas, caso as análises tivessem partido dos outros 174 artigos (pertencentes a 109 diferentes periódicos), ou ainda de bases de dados mais extensas, no limite representando o censo do campo de conhecimento. Uma resposta positiva a essa pergunta reafirmaria a validade e a riqueza de trabalhos como o aqui proposto, por permitir ao pesquisador mapear o seu campo de conhecimento de maneira mais rápida e objetiva.

\section{REFERÊNCIAS}

ALONSO-ALMEIDA, Maria del Mar; BREMSER, Kerstin.

Strategic responses of the Spanish hospitality sector to the financial crisis. International Journal of Hospitality

Management, UK, v. 32, p. 141-148, 2013.

ANGELO, C. F.; FAVERO, L. P. L.; EUNNI, R.V. Impact of loyalty programs on customer retention: evidence from the retail apparel industry in Brazil. Anais Eletrônicos. CD-ROM, AGB: IALBSS, Washington, D.C., 2007.

ARMSTRONG, Arthur; HAGEL, John. The real value of online communities. Harvard Business Review, USA, v. 74, n. 3, p. 134-141, 1996.

BAUMGARTNER, Hans; PIETERS, Rik. The Structural Influence of Marketing Journals: a Citation Analysis of the Discipline and Its Subareas Over Time. Journal of Marketing, USA, v. 67, p. 123-139, Apr. 2003.

BERMAN, Barry. Developing an Effective Customer Loyalty Program. California Management Review, USA, v. 49, n. 1, p.123-148, 2006.

BRANDES, Ulrik; KENIS, Patrick; WAGNER, Dorothea. Communicating centrality in policy network drawings. IEEE Transactions on Visualization and Computer Graphics, USA, v. 9, n. 2, p. 241-253, 2003.

BUTTS, C. T. Network: a package for managing relational data in R. Journal of Statistical Software, USA, v. 24, n. 2, 36 p., 2008.

BUTTS, C. T; HANDCOCK, M. S; HUNTER, D. R.

Network: classes for relational data. R package version 1.6. Irvine, 2011. Disponível em: < http://statnet.org> . Acesso em: 28 jan. 2011.

CAPIZZI, Michael T.; FERGUSON, Rick. Loyalty Trends for the Twenty-first Century. Journal of Consumer

Marketing, UK, v. 22, n. 2, p. 72-80, 2005.

CHANDON, Pierre; WANSINK, Brian; GILLES, Laurent.

A Benefit Congruency Framework of Sales Promotion Effectiveness. Journal of Marketing, USA, v. 64 n. 4, p. 65-81, 2000. 
DAY, George S. A two-dimensional concept of brand loyalty. Journal of Advertising Research, UK, v. 9, n. 3, p. 29-35, 1969.

DAY, George S. A Empresa orientada para o mercado: compreender, atrair e manter clientes valiosos. São Paulo: Bookman, 2001.

DICK, Alan; BASU, Kunal. Customer loyalty: toward an integrated conceptual framework. Journal of the Academy of Marketing Science, USA, v. 22, n. 2, p. 99-113, 1994.

FOWLER, R. J. Database Implementation for the TIN data structure. Technical Report 11, Dept. of Geography, Simon Fraser Univ, B. C, Burnaby, 1976.

FRANCISCO, Eduardo de R. RAE-Eletrônica: exploração do acervo à luz da bibliometria, geoanálise e redes sociais. Revista de Administração de Empresas, São Paulo, v. 51, n. 3, p. 280-306, 2011.

FRANK, Ronald E. Conclates of buying behavior for grocery products. Journal of Marketing, USA, v. 31, p. 48-53, October 1967.

GARFIELD, E. Citation indexing: its theory and application in science, technology and humanities. New York: Wiley, 1979.

GARFIELD, E. The Significant Scientific Literature Appears in a Small Core of Journals. The Scientist, USA, v. 10, n. 17, p. 13-16, 1996.

HANNEMAN, R. A; RIDDLE, M. Introduction to social network methods. Riverside: University of Califórnia, 2005. Disponível em: < http://faculty.ucr.edu/ hanneman/ nettext/index.html>. Acesso em: 28 jan. 2011.

HARDESTY, David M.; BEARDEN, William O. Consumer Behavior and Retailing. Journal of Retailing,

Netherlands, v. 85, n. 3, p. 239-244, 2009.

HLAVINKA, K.; SULLIVAN, J. The Billion Member March: the 2011 COLLOQUY Loyalty Census. Colloquy. Milford, OH: April, 2011.

HUANG, Leo. Exploring the determinants of e-loyalty among travel agencies. The Services Industries Journal, UK, v. 28, n. 2, p. 239-254, 2008.
JOHNSON, Grace; BRUNER, Gordon; KUMAR, Anand. Interactivity and its facets revisited. Journal of Advertising, USA, v. 35, n. 4, p. 35-52, 2006.

LEVY, Michael; WEITZ, Barton A. Retailing

Management. $6^{\text {th }}$ ed. Oak Brook, IL: McGraw-Hill/Irwin, 2007.

LIANG, Dapeng; MA, Zhenzhong; QI, Liyun. Service quality and customer switching behavior in China's mobile phone service sector. Journal of Business Research, In Press, Corrected Proof. Disponível em: <http://dx.doi. org/10.1016/j.jbusres.2012.03.012>. Acesso em: 9 jun. 2012.

MARQUES, Licione; BRASIL, Vinícius S. Validação de um Modelo de Lealdade do Estudante com Base na Qualidade do Relacionamento. In: ENCONTRO DA ASSOCIAÇÃO NACIONAL DE PÓS-GRADUAÇÃO EM ADMINISTRAÇÃO, 32., Rio de Janeiro: Enanpad, 2008. Anais... Rio de Janeiro, Enampad, 2008.

MCMULLAN, Rosalind. A multiple-item scale for measuring customer loyalty development. Journal of Services Marketing, UK,v. 19, n. 7, p. 470-481, 2005.

MCMULLAN, Rosalind; GILMORE, Audrey. The conceptual development of customer loyalty measurement: a proposed scale. Journal of Targeting, Measurement and Analysis for Marketing, UK, v. 11, n. 3, p. 230-243, 2003.

MELNYK, Valentyna; OSSELAER, Stijn M. J. Make me special: Gender differences in consumers' responses to loyalty programs. Marketing Letters, USA, v. 23, n. 3, p. 545-559, 2012.

MOODY, J. The structure of a social science collaboration network: disciplinary cohesion from 1963 to 1999.

American Sociological Review, USA, v. 69, n. 2, p. 213-238, 2004.

NARAYANDAS, Das. Building Loyalty in Business Markets. Harvard Business Review, USA, p. 131-39, Sept. 2005.

NEWMAN, M. The structure of scientific collaboration networks. Proceedings of National Academic

Sciences, USA, v. 98, n. 2, p. 404-409, 2001. 
NUNES, Joseph C.; DRÈZE, Xavier. Your Loyalty Program Is Betraying You. Harvard Business Review, USA, p. 124-31, April, 2006.

OLIVER, Richard L. Whence customer loyalty. Journal of Marketing, USA, v. 63, special issue, p. 33-44, 1999.

OLIVEIRA, Braulio A. C.; TOLEDO, Geraldo L.; IKEDA, Ana A. Fidelização e valor uma interdependência inequívoca. In: SEMEAD 7. USP/FEA/PPGA, São Paulo, 2004. Anais... São Paulo: São Paulo, 2004.

PAN, Yue; SHENG, Simon; XIE, Frank T. Antecedents of customer loyalty: An empirical synthesis and reexamination. Journal of Retailing and Consumer Services, UK, v. 19, p. 150-158, 2012.

\section{PORTAL FOLHA DE SÃO PAULO. Programa de}

fidelidade cresce na classe C. [2013]. Disponível em: <http:/www1.folha.uol.com.br/mercado/1252461programa-de-fidelidade-cresce-na-classe-c.shtml $>$. Acesso em: 30 mar. 2013.

RABBANEE, Fazlul; RAMASESHAN, B.; WU, Chen; VINDEN, Amy. Effects of store loyalty on shopping mall loyalty. Journal of Retailing and Consumer Services, UK, v. 19, p. 271-278, 2012.

ROCHA, Thelma; VELOSO, André. A hora da recompensa. São Paulo: Editora Cobra, 1999.

SIRDESHMUKH, Deepak; SINGH, Jagdip; SABOL, Berry. Consumer Trust, Value, and Loyalty in Relational Exchanges. Journal of Marketing, USA, v. 66, n. 1, p. 15-37, 2002.

\section{SPINAK, E. Diccionario enciclopédico de}

bibliometría, cienciometría e informetría. Montevideo: UNESCO, 1996.

SHUKLA, Paurav. Impact of contextual factors, brand loyalty and brand switching on purchase decisions.

Journal of Consumer Marketing, UK, v. 26, n. 5, p. 348-357, 2009.

TOUFAILY, Elissar; RICARD, Line; PERRIEN, Jean. Customer loyalty to a commercial website: descriptive meta-analysis of the empirical literature and proposal of an integrative model. Journal of Business Research, In Press, Corrected Proof. Disponível em: <http://dx.doi.org/10.1016/j.

jbusres.2012.05.011>. Acesso em: 11 jul. 2012
YOO, Myongjee; BAI, Billy. Customer loyalty marketing research: a comparative approach between hospitality and business journals. International Journal of Hospitality Management, UK, v. 33, p. 166-177, 2013.

ZEITHAML, Valarie A. Service quality, profitability, and the economic worth of customers: what we know and what we need to learn. Journal of the Academy of Marketing Science, USA, v. 28, n. 1, p. 67-85, 2000. 